\title{
Die Bedienung des Automatens durch den Mensch. Deklination der schwachen Maskulina als Zweifelsfall
}

\author{
Rolf Thieroff (Osnabrück)
}

\begin{abstract}
In New High German the following five declension classes of the noun are distinguished: 1) the feminine declension class (comprising all feminine nouns), 2) the so-called $s$-class (with masculine and neuter nouns), 3), the class of strong nouns (masculine and neuter nouns), 4) the mixed class (masculine and neuter nouns) and 5) the class of weak nouns (masculine nouns only). In the spoken register and sometimes even in the written register, speakers of German tend to inflect certain weak nouns according to the mixed or even to the strong class. The present paper consists of two major parts. In the first part, I give an account of how the inflection of weak nouns is treated in two dictionaries giving advice to readers being in doubt about the correct form. In the second part, I investigate possible reasons for the transition of nouns from the weak to the mixed or to the strong class. I argue that the weak declension differs considerably from all other declension classes in that the accusative and the dative of the singular are marked by a case suffix (absent in all other classes), that only in this class the genitive of the singular is not marked by the suffix $-s$ and that only in the weak class there are two different forms for the accusative and the dative singular, depending on the syntactic context. Accordingly, speakers' doubts about the correct inflection of weak nouns are explained by the deviance of the weak class from all other declension classes of Modern
\end{abstract} German.

\section{$1 \quad$ Einleitung}

Es ist allgemein bekannt, dass bei einer Anzahl von Substantiven, die ursprünglich nach der schwachen Deklination flektieren, eine Tendenz besteht, den Akkusativ und den Dativ Singular nicht mehr zu markieren, d.h. ohne Kasussuffix zu bilden, oder, wie es der Duden Band 9, Richtiges und gutes Deutsch, formuliert:

Es besteht eine starke Neigung, bei schwach gebeugten maskulinen Substantiven im Dativ und Akkusativ Singular die Deklinationsendung abzuwerfen und die Substantive dadurch zu starken zu machen. (Duden 2001: 859)

Einmal davon abgesehen, dass ein Substantiv allein durch das "Abwerfen" der "Deklinationsendung" im Akkusativ und Dativ Singular noch lange nicht zu einem starken gemacht wird, ist es richtig und sinnvoll, dieses Problem in einem Werk über sprachliche Zweifelsfälle zu behandeln. Dass hier Zweifel bei Sprachteilhabern bestehen, steht außer Frage. Dabei geht es 
nicht nur um die Frage, wie die Akkusativ- oder Dativform eines schwachen Substantivs zu bilden ist, sondern auch Fragen nach der Genitivform werden gestellt (z.B. "Wie lautet der Genitiv von Hase, des Hasen oder des Hasens?"), und gelegentlich geraten sogar starke Substantive in den Verdacht, zur schwachen Deklination zu gehören. So wurde im Sprachdienst im Jahre 1996 die Frage gestellt, ob Elch stark oder schwach dekliniert wird, und das Substantiv Autor, das eigentlich zur gemischten Deklination gehört, findet man sogar relativ oft schwach dekliniert. Hier einige Belege aus jüngerer Zeit:

(1) Mike gibt seinem Pilot das Zeichen zur Beschleunigung.

(Frankfurter Allgemeine Zeitung, 15.10.2001, S. 2)

(2) Wird die Selbstverwaltung damit zum bloßen Erfüllungsgehilfe der Legislative?

(Frankfurter Allgemeine Zeitung, 06.04.1999, S. 19)

(3) Sicherheitsdenken ist die älteste Überlebenstechnik des Menschens, nur haben wir heute mehr zu bedenken als Nüsse zu sammeln und möglichst einen großen Bären zu erlegen.

(Die Welt, 03.01.2000, S. 31)

(4) Dem Autoren eines Buches über die Satanischen Verse von Salman Rushdie wurde vorgeworfen, er sei gegen die von Chomeini verhängte Fatwah gegen den britischen Autor. (Frankfurter Allgemeine Zeitung, 18.12.2000, S. 5)

(5) VW [...] hat den Platzhirschen Mercedes-Benz verdrängt.

(Berliner Morgenpost, 05.02.2003)

Zweifel bestehen also, und "falsche" Formen kommen tatsächlich vor, sogar in einer Zeitung wie der FAZ, die nicht gerade für sprachliche Schludrigkeiten bekannt ist.

Im folgenden zweiten Teil der vorliegenden Arbeit zeige ich zunächst, wie das Problem in zwei Zweifelsfälle-Wörterbüchern behandelt wird, nämlich in Duden Band 9, Richtiges und gutes Deutsch, 5. Auflage, 2001 (im Folgenden kurz Duden; Abschnitt 2.1) und im soeben erschienenen Wahrig Band 5, Fehlerfreies und gutes Deutsch, 2003 (im Folgenden kurz Wahrig; Abschnitt 2.2). Im dritten Teil der Arbeit gehe ich dann den Gründen für die Übergänge von der schwachen zur gemischten bzw. zur starken Deklinationsklasse nach (Abschnitt 3). Dabei wird sich zeigen, dass die Darstellung im Wahrig die gegenüber jener im Duden angemessenere ist.

\section{Behandlung der schwachen Maskulina in Zweifelsfälle-Wörterbüchern}

\subsection{Richtiges und gutes Deutsch}

Im Duden lautet die Überschrift, unter der das Problem behandelt wird: "Nicht anerkannte Unterlassung der Deklination" (Duden 2001: 859).

Der Abschnitt beginnt mit dem eingangs zitierten Satz, gefolgt von ein paar Beispielen, die in der folgenden Form präsentiert werden:

(6) den Gendarm (Fallada; statt: den Gendarmen); den Kurfürst (W. Schäfer; statt: den Kurfürsten); einen ausgezeichneten Geck (Hofmannsthal; statt: Gecken); [...]

(Duden 2001: 859) 
Man sieht also sogleich, dass auch anerkannte Schriftsteller im fraglichen Bereich "Fehler" machen. Freilich muss man Fallada und Hofmannsthal zugute halten, dass sie nicht die Möglichkeit hatten, im Duden nachzuschlagen, wie man Gendarm und Kurfürst "richtig" dekliniert.

Weiter heißt es im Duden (ebd.): "Die starken Formen werden dann auch auf den Genitiv übertragen", gefolgt von den Beispielen in (7), wo sich zeigt, dass auch Gerhart Hauptmann den Duden gut hätte gebrauchen können.

(7) die Mütze des Bubs (statt: des Buben); das Auftreten dieses Gecks (statt: dieses Gecken); mit des Markgrafs (statt: des Markgrafen) Weib (G. Hauptmann)

(Duden 2001: 860)

Auf diese Beispiele folgt der Satz: "Hier handelt es sich also nicht nur um Unterlassung der Beugung, sondern um einen Wechsel der Deklinationsklasse, der aber fast ausschließlich den Singular betrifft. Im Plural bleiben diese Substantive [...] schwach." (Duden 2001: 860)

Während das alles "nicht korrekt" ist, gibt es für den Duden immerhin noch den folgenden Fall:

Bei einigen Substantiven, die früher nur schwach gebeugt wurden, hat sich allerdings die starke Beugung im Singular neben der schwachen durchgesetzt:

der Ahn, Genitiv: des Ahns oder des Ahnen; der Untertan, Genitiv: des Untertans, auch: des Untertanen; der Bauer, Genitiv: des Bauern, selten: des Bauers; der Spatz, Genitiv: des Spatzen, auch: des Spatzes; der Oberst, Genitiv: des Obersten oder des Obersts, Plural: die Obersten, seltener: die Oberste. (Duden 2001: 860)

Nach dieser Einleitung folgen dann drei Listen mit Wörtern, bei denen die "Unterlassung der Deklination" nicht korrekt ist. Das sieht wie folgt aus:

Deutsche Wörter und Lehnwörter: Bei den folgenden deutschen oder entlehnten Substantiven ist die Unterlassung der Deklination nicht korrekt:

des Bärs, dem, den Bär statt: des Bären, dem, den Bären; [...]; des Finks, dem, den Fink, statt: des Finken, dem, den Finken; des Gecks, dem, den Geck, statt: des Gecken, dem, den Geck; dem, den Held, statt: dem, den Helden; dem, den Hirt, statt: dem, den Hirten; dem, den Mensch, statt: dem, den Menschen, [...] (Duden 2001: 860)

Es folgen noch acht weitere, also insgesamt 14 Fälle. Weiter heißt es:

Fremdwörter: Bei den folgenden fremden Substantiven ist die Unterlassung der Deklination nicht korrekt:

des Automats, dem, den Automat statt: des Automaten, dem, den Automaten; [...]; dem, den Diplomat statt: dem, den Diplomaten; des Elefants, dem, den Elefant statt: des Elefanten, dem, den Elefanten; dem, den Exponenten statt dem, den Exponent; [...] (Duden 2001: 860f.)

Hier folgen 17 weitere, also insgesamt 21 Fälle. Und schließlich: 
Bei einer Reihe von Fremdwörtern wird die Deklination häufig unterlassen, wenn sie als Rangbezeichnung, Titel oder Berufsbezeichnung, und in diesen Fällen oft in Verbindung mit dem Namen, gebraucht werden:

dem, den Architekt statt: dem, den Architekten; dem, den Dirigent statt: dem, den Dirigenten; dem, den Dozent statt: dem, den Dozenten; dem, den Drogist statt dem, den Drogisten; [...][...] Diese Unterlassung der Deklination ist nicht korrekt. (Duden 2001: 861)

Es folgen zehn weitere Substantive, so dass hier also insgesamt 14 Fälle genannt werden.

Die Aussagen des Duden lassen also an Eindeutigkeit nichts zu wünschen übrig. Der Nutzer erhält, zumindest was diese 49 Wörter betrifft, eine klare Antwort auf die Frage, wie die Wörter zu deklinieren seien: Die (vorgeblich) "starke" (tatsächlich: gemischte) Deklination ist falsch, die schwache ist richtig.

Allerdings ist die ganze Darstellung nicht unproblematisch, und zwar hinsichtlich der folgenden vier Punkte:

1. Die gesamte Darstellung ist recht unübersichtlich. Insbesondere fällt auf, dass mal nur der Akkusativ und der Dativ Singular, mal Akkusativ, Dativ und Genitiv Singular und mal zusätzlich auch noch der Plural aufgeführt werden. So wird bei den Beispielen im ersten Zitat bei Bär, Fink und Geck der Genitiv genannt, bei Held, Hirt und Mensch dagegen nicht, und in dieser bunten Abwechslung geht es bei den folgenden, hier nicht zitierten Beispielen weiter. Genau so ist es bei den Beispielen im zweiten Zitat: Automat wird mit Genitiv, Diplomat ohne, Elefant wieder mit, Exponent wieder ohne Genitiv aufgeführt, und so geht es weiter, ohne dass eine Begründung dafür angegeben würde. Lediglich in der dritten Liste kommen keine Genitivformen vor.

2. Falsch ist (wie schon angedeutet) die Aussage, dass bei dem Typ den Bär, dem Bär, des Bärs, die Bären ein Wechsel in die starke Deklinationsklasse vorläge. Solange nämlich der Plural auf - $(e) n$ gebildet wird, liegt das Muster der gemischten Deklination vor, die genau so auch unter dem Stichwort Substantiv. Die Deklinationsarten im selben Buch zu finden ist (Duden 2001: 806).

3. Der Duden ist hier (anders als in vielen anderen Fällen) äußerst normativ und von erstaunlicher Rigidität. Die Möglichkeit, dass hier - sogar gut begründbare - Sprachwandelphänomene vorliegen könnten, wird nicht in Betracht gezogen.

4. Es gibt (im Gegensatz zu einer Reihe von anderen Artikeln) keinerlei Erklärungen für die Übergänge zur gemischten und (sehr selten) zur starken Deklination.

\subsection{Fehlerfreies und gutes Deutsch}

Die Antwort auf die Frage, ob und gegebenenfalls wie man es besser als der ehrwürdige Zweifelsfälle-Duden machen kann, scheint mir der soeben erschienene Wahrig Band 5, Fehlerfreies und gutes Deutsch (im Folgenden kurz Wahrig) zu geben. Im Wahrig heißt es, ebenfalls in einem einleitenden Artikel zu dem Problem: 
Einige Substantive tendieren dazu, von der schwachen in die starke Deklinationsklasse zu wechseln. Es gibt drei Stufen des Übergangs.

1. Stufe: Akkusativ und Dativ Singular werden ohne die Endung -en gebildet. Der Genitiv Singular und der Plural behalten hingegen die Endung -en bei

den/dem Mensch statt den/dem Menschen; des Menschen; die Menschen

2. Stufe: Die schwache Endung -en im Genitiv Singular wird zusätzlich durch die starke Endung $-(e) s$ ersetzt. Lediglich im Plural bleibt die Endung -en erhalten. Substantive dieser Stufe können also wie Substantive der gemischten Deklinationsklasse flektiert werden:

den/dem Mohr statt den/dem Mohren;

des Mohrs statt des Mohren; die Mohren

3. Stufe: Die Pluralendung -en kann durch die Pluralendung -e der starken Deklinationsklasse ersetzt werden. Substantive dieser Stufe können also auch stark flektiert werden:

den/dem Magnet statt den/dem Magneten;

des Magnets statt des Magneten;

die Magnete statt die Magneten).

Übergänge von der schwachen zur starken Deklination kommen ausschließlich bei Substantiven vor, die im Nominativ Singular auf Konsonant enden, nicht bei solchen, die auf unbetontes - $e$ auslauten (wie etwa der Bote). In der Schriftsprache sollte in der Regel die schwache Deklination beibehalten werden, die starke Deklination gilt bei den meisten Substantiven als umgangssprachlich. (Wahrig 2003: 316)

Es folgen dann drei weitere Artikel, in denen diese drei Fälle genauer beschrieben werden. Die im Duden aufgeführten Wörter und einige mehr werden dabei jeweils einer der drei Gruppen zugeordnet. So heißt es unter der Überschrift "Wegfall von Akkusativ/Dativ Singular-en bei schwachen Maskulina":

Bei einigen Maskulina der schwachen Deklination kommen im Akkusativ und Dativ Singular neben den schwachen Formen auf -en auch Formen ohne diese Endung vor. Diese Formen gelten als umgangssprachlich, sie sollten in der Schriftsprache vermieden werden.

$\begin{array}{lll} & \text { Singular } & \text { Plural } \\ \text { Nom. } & \text { der Mensch } & \text { die Menschen } \\ \text { Akk. } & \text { den Menschen / den Mensch } & \text { die Menschen } \\ \text { Dat. } & \text { dem Menschen / dem Mensch } & \text { den Menschen } \\ \text { Gen. } & \text { des Menschen } & \text { der Menschen }\end{array}$

Wie Mensch verhalten sich auch die folgenden einsilbigen Maskulina:

Fürst, Graf, Held, Hirt, Prinz, Tor, Zar

Darüber hinaus kann bei einer Anzahl von Fremdwörtern, die sämtlich Personen bezeichnen,

Akkusativ- und Dativ-en weggelassen werden. Auch hier gilt, dass die Unterlassung der

Deklination in der Schriftsprache vermieden werden sollte:

der Architekt, Diplomat, Dirigent, Dramaturg, Drogist, Fabrikant, Fotograf, [...]

Auch einige Substantive, die keine Personen bezeichnen, gehören zu dieser Gruppe:

der Brillant, Exponent, Hydrant, Obelisk

ISSN 1615-3014 
Die Substantive Bursche und Ochse können nur dann ohne Akkusativ-/Dativ- $n$ stehen, wenn ihnen zugleich auch das auslautende unbetonte $-e$ fehlt:

den/dem Bursch (neben den/dem Burschen);

den/dem Ochs (neben den/dem Ochsen)

(Wahrig 2003: 316f.)

Die beiden folgenden Artikel sind analog aufgebaut. Substantive wie Mohr, so heißt es im folgenden Artikel, können entweder schwach oder gemischt flektiert werden. Das wird wiederum mittels eines Paradigmas veranschaulicht, wie in (8).

Paradigma von Mohr (Wahrig 2003: 317)

$\begin{array}{lll} & \text { Singular } & \text { Plural } \\ \text { Nom. } & \text { der Mohr } & \text { die Mohren } \\ \text { Akk. } & \text { den Mohren /den Mohr } & \text { die Mohren } \\ \text { Dat. } & \text { dem Mohren/dem Mohr } & \text { den Mohren } \\ \text { Gen. } & \text { des Mohren /des Mohrs } & \text { der Mohren }\end{array}$

Wiederum folgen Listen von Wörtern, die so flektiert werden, getrennt nach Substantiven deutschen Ursprungs und Fremdwörtern, und zusätzlich wird $B u b$ aufgeführt, für das analog dasselbe gilt wie für Bursch und Ochs.

Und schließlich folgen diejenigen Wörter, die tatsächlich schwach und stark dekliniert werden können, wiederum veranschaulicht mittels eines Paradigmas, wie in (9):

(9) Paradigma von Greif (Wahrig 2003: 318)

$\begin{array}{lll} & \text { Singular } & \text { Plural } \\ \text { Nom. } & \text { derGreif } & \text { die Greifen / die Greife } \\ \text { Akk. } & \text { denGreifen / den Greif } & \text { die Greifen / die Greife } \\ \text { Dat. } & \text { dem Greifen / dem Greif } & \text { den Greifen /den Greifen } \\ \text { Gen. } & \text { des Greifen /des Greifs } & \text { der Greifen /der Greife }\end{array}$

Anders als im Duden ist hier also nicht von "richtig" und "falsch" die Rede; dennoch werden auch hier überwiegend die schwachen Formen empfohlen. Näherer Erklärungen, wie es zu diesen Übergängen kommt, enthält sich der Wahrig ebenso wie der Duden. Dass hier kaum etwas erklärt wird, liegt allerdings nicht daran, dass man dazu nichts sagen könnte oder nichts darüber wüsste, sondern daran, dass die Gründe ziemlich komplex sind und mehrere Faktoren eine Rolle spielen, und das alles darzulegen wäre für ein Zweifelsfälle-Nachschlagewerk kaum angemessen. Ich will im Folgenden versuchen, die Gründe für die Übergänge von der schwachen zur gemischten (und in Ausnahmefällen zur starken) Deklination kurz darzustellen. 


\section{Gründe für die Deklinationsklassenübergänge}

\subsection{Prototypen}

Köpcke (1995) hat nachgewiesen, dass es zwei Prototypen für die schwach deklinierten Maskulina gibt, die die folgenden Eigenschaften haben. Schwache Maskulina des Prototyps I haben das semantische Merkmal [+ human], lauten auf Schwa aus, haben Pänultimabetonung und sind mehrsilbig. Schwache Maskulina des Prototyps II haben ebenfalls das Merkmal [+ human], haben Ultimabetonung und sind gleichfalls mehrsilbig (Köpcke 1995: 168).

In einer Tabelle zeigt Köpcke bezüglich des Prototyps I, wie weit die vorkommenden Typen von schwachen Maskulina von diesem Prototyp entfernt sind:

(10) Schwache Maskulina des Prototyps I (Köpcke 1995:170)

\begin{tabular}{|c|c|c||c|c|c|}
\hline mehrsilbig & $\begin{array}{l}\text { Penultima- } \\
\text { betonung }\end{array}$ & Schwa & [+ menschl. $]$ & [+ belebt $]$ & [- belebt $]$ \\
\hline+ & + & + & $\begin{array}{c}\text { I } \\
\text { Matrose }\end{array}$ & $\begin{array}{c}\text { II } \\
\text { Oktopode }\end{array}$ & $\begin{array}{c}\text { III } \\
\text { Gedanke }\end{array}$ \\
\hline- & + & + & $\begin{array}{c}\text { IV } \\
\text { Kurde }\end{array}$ & $\begin{array}{c}\text { V } \\
\text { Falke }\end{array}$ & $\begin{array}{c}\text { VI } \\
\text { Glaube }\end{array}$ \\
\hline- & - & - & $\begin{array}{c}\text { VII } \\
\text { Mensch }\end{array}$ & $\begin{array}{c}\text { VIII } \\
\text { Bär }\end{array}$ & $\begin{array}{c}\text { IX } \\
-\end{array}$ \\
\hline
\end{tabular}

Das prototypische schwache Maskulinum ist also eines wie Matrose in Zelle I. Die Prototypizität nimmt sowohl von links nach rechts als auch von oben nach unten ab. Dem entspricht grob die Anzahl der Wörter, die in den einzelnen Feldern vorkommen. So gibt es laut Köpcke etwa 280 Wörter vom Typ Matrose, aber nur 5 vom Typ II und nur ein einziges vom Typ III. Und den 280 Wörtern von Typ I stehen ca. 70 von Typ IV und ca. 15 von Typ VII gegenüber. Zu erwarten ist nun, dass die am wenigsten prototypischen Substantive am ehesten die Tendenz haben, die Klasse der schwachen Maskulina zu verlassen, und genau das ist der Fall. Die Substantive in III und VI tendieren dazu, den Nominativ Singular mit $n$ zu bilden, was dazu führt, dass das $n$ als Teil des Stamms reanalysiert wird, und damit flektieren sie stark - vgl. (11). ${ }^{1}$

\footnotetext{
${ }^{1}$ Dass dieser Übergang nicht für alle Substantive vom Typ Funke/Funken gilt, zeigt Joeres (1996). Darauf kann hier nicht näher eingegangen werden.
} 
(11) Übergang von der schwachen zur gemischten Deklination bei Substantiven vom Typ Funke(n)

Schwache Flexion

\begin{tabular}{|c|c|c|}
\hline & & $\mathrm{Sg}$ \\
\hline Nom & Funke & - \\
\hline Akk & & $-n$ \\
\hline Dat & & $-n$ \\
\hline Gen & & $-n$ \\
\hline
\end{tabular}

Starke Flexion durch Hinzufügung von $n$ im NomSg

$\begin{array}{rll} & \text { Sg } & \text { Pl } \\ \text { Funken } & - & - \\ & - & - \\ & - & - \\ & -S & -\end{array}$

Von den Substantiven der Gruppe VIII können drei auch gemischt flektiert werden, nämlich Bär, Fink und Spatz, und zwei stark, nämlich Greif und Pfau. Dramatischer (und nicht ganz im Einklang mit Köpckes Voraussagen) sind die Tendenzen bei VII. Laut Köpcke gibt es "ca. 15" Wörter in dieser Gruppe. Wenn wir einmal die Sonderfälle Bub und Bursch außer acht lassen, dann gibt es exakt 15 Einsilbler mit dem Merkmal [+ human], die Tendenzen zur starken Flexion haben und die sich wie folgt auf die drei Gruppen aus Wahrig 2003 verteilen:

(12) Einsilbler, [+ human]

1. Akk/DatSg ohne -en (GenSg -en):

Christ, Fürst, Graf, Held, Hirt, Mensch, Prinz, Tor, Zar; Schenk

10

2. gemischte Deklination:

Ahn, Geck, Mohr, Narr

4

3. starke Deklination:

Gnom

Die Zellen VII und VIII sind also erstens schwach besetzt, und zweitens gibt es ganz eindeutig einen Trend, diese Zellen gänzlich zu räumen, besonders deutlich bei Zelle VII. Dass gerade die Substantive in (12) nicht mehr von allen Sprechern schwach flektiert werden und in die Klasse der gemischten übertreten, dass hier ein echtes Sprachwandelphänomen vorliegt, kann also gut begründet werden. Substantive, die so wie Mensch gebaut sind, flektieren normalerweise einfach nicht schwach, d.h. sie sind Ausnahmen im System, und diese Ausnahmen werden offensichtlich zugunsten einer größeren Systematizität abgebaut. Es ist also zu erwarten, dass diese 15 Substantive in absehbarer Zeit endgültig in die Klasse der gemischt flektierenden (bzw. im Falle von Gnom der stark flektierenden) Substantive übertreten, und ein solcher Übertritt wäre aus systemlinguistischer Sicht eher zu begrüßen denn zu verhindern.

Auch bei den Substantiven, die dem Prototyp II zuzurechnen sind, gibt es eine Reihe von Ausnahmen, die nicht richtig dazu gehören. Am untypischsten sind natürlich diejenigen schwachen Maskulina, die etwas Unbelebtes bezeichnen. Allerdings sind die Ausreißertendenzen hier nicht so deutlich wie beim Prototyp I. Köpcke kommt auf 52 schwache Maskulina, die Nicht-Belebtes bezeichnen, und nur acht werden in Duden und/oder Wahrig erwähnt, nämlich die in (13). 
(13) mehrsilbige schwache Maskulina mit Ultimabetonung, [- anim]

1. Akk/DatSg ohne -en (GenSg-en):

Brillant, Exponent, Hydrant, Obelisk

2. gemischte Deklination:

Automat, Komet, Planet

3. starke Deklination:

Magnet

Hinzu kommen noch zwei weitere, die zwar nicht Unbelebtes, aber auch nicht Menschliches bezeichnen, nämlich Elefant und Leopard, die also ebenfalls dazu tendieren, nicht schwach zu flektieren, weil sie nicht prototypisch sind.

Ein Problem für eine Erklärung à la Köpcke ist allerdings die Tatsache, dass es mindestens 25 und mit Sicherheit noch mehr Maskulina des Typs 'mehrsilbig, Ultimabetonung' gibt, die menschliche Lebewesen bezeichnen und dennoch die Tendenz haben, im Akkusativ und Dativ ohne -en, u.U. auch im Genitiv mit $-s$ benutzt zu werden (Architekt, Dirigent, Dozent, Drogist im Duden-Zitat). Das sind also prototypische schwache Maskulina, und trotzdem werden sie nicht konsequent von allen Sprechern schwach flektiert. Es muss also noch andere Gründe geben für die Tendenz zur starken Flexion.

\subsection{Schwache Maskulina und die anderen Deklinationsklassen}

Man kann mindestens die folgenden fünf Deklinationsklassen mit verschiedenen Subklassen unterscheiden:

1. Feminine Deklinationsklasse, 3 Subklassen

\begin{tabular}{|c|c|c|c|c|c|c|c|c|}
\hline & & $\mathrm{Sg}$ & $\mathrm{Pl}$ & & $\mathrm{Sg}$ & $\mathrm{Pl}$ & & $\mathrm{Sg}$ \\
\hline Nom & Oma & - & $-\mathrm{s}$ & Frau & - & -en & Hand & - \\
\hline Akk & & - & $-\mathrm{s}$ & & - & -en & & - \\
\hline Dat & & - & $-\mathrm{s}$ & & - & -en & & - \\
\hline Gen & & - & $-\mathrm{s}$ & & - & -en & & - \\
\hline
\end{tabular}

2. Gemischte Deklinationsklasse

\begin{tabular}{|c|c|c|}
\hline & & $\mathrm{Sg}$ \\
\hline Nom & Staat & - \\
\hline Akk & & - \\
\hline Dat & & - \\
\hline Gen & & -es \\
\hline
\end{tabular}

3. Starke Deklinationsklasse, 4 Subklassen

\begin{tabular}{|c|c|c|c|c|c|c|c|c|c|c|c|}
\hline & & $\mathrm{Sg}$ & $\mathrm{Pl}$ & & $\mathrm{Sg}$ & $\mathrm{Pl}$ & & $\mathrm{Sg}$ & $\mathrm{Pl}$ & & $\mathrm{Sg}$ \\
\hline Nom & Berg & - & $-e$ & Kind & - & -er & Vater & - & $\ddot{-}$ & Wagen & - \\
\hline Akk & & - & $-e$ & & - & -er & & - & $\ddot{-}$ & & - \\
\hline Dat & & - & -en & & - & -ern & & - & ${ }^{\prime}-n$ & & - \\
\hline Gen & & -es & $-e$ & & -es & -er & & $-\mathrm{S}$ & $"$ & & $-\mathrm{s}$ \\
\hline
\end{tabular}


4. s-Deklinationsklasse

$\begin{array}{llll} & & \text { Sg } & \text { Pl } \\ \text { Nom } & \text { Opa } & - & -s \\ \text { Akk } & & - & -s \\ \text { Dat } & & - & -s \\ \text { Gen } & & -s & -s\end{array}$

5. Schwache Deklinationsklasse, 2 Subklassen

\begin{tabular}{|c|c|c|c|c|c|}
\hline & & $\mathrm{Sg}$ & $\mathrm{Pl}$ & & $\mathrm{Sg}$ \\
\hline Nom & Mensch & - & -en & Name & - \\
\hline Akk & & -en & -en & & $-n$ \\
\hline Dat & & -en & -en & & $-n$ \\
\hline Gen & & -en & -en & & $-n s$ \\
\hline
\end{tabular}

Wenn wir nun die Deklinationsklassen 1 bis 4 miteinander vergleichen, können wir drei frappierende Gemeinsamkeiten dieser vier Klassen feststellen.

Erstens: In keiner dieser Deklinationsklassen wird der Akkusativ oder der Dativ Singular mit einem Suffix markiert, d.h. es ist für die Deklination des Substantivs im Deutschen normal, dass Akkusativ und Dativ nicht markiert werden. Die Markierung von Akkusativ und Dativ Singular mittels Suffix ist ganz offensichtlich nicht normal, jedenfalls nicht bei Substantiven. Wer also den Mensch und dem Architekt sagt, der tut nichts anderes, als Mensch und Architekt wie ein normales Substantiv zu behandeln. Kann und soll man das verbieten?

Zweitens: Bei allen Nicht-Feminina (zu denen die schwachen Maskulina ja gehören) ist der Genitiv Singular immer formal von Akkusativ und Dativ unterschieden. Das gilt sogar für eine Subklasse der Schwachen, nämlich für den Typ Name! Offensichtlich ist es normal, dass der Genitiv nicht mit Akkusativ und Dativ homonym ist. Tatsächlich kann man zeigen, dass es aus syntaktischen Gründen in der Tat äußerst wichtig ist, dass der Genitiv Singular formal möglichst immer von allen anderen Kasus unterschieden wird. Wer also den Christ, dem Christ und des Christen, oder den Diplomat, dem Diplomat und des Diplomaten sagt, der befolgt die allgemeine Regel, dass Maskulina sich im Deutschen im Genitiv Singular von den anderen Kasus zu unterscheiden haben, d.h. er tut nichts anderes, als Christ und Diplomat wie normale Substantive zu behandeln. Kann und soll man das verbieten?

Drittens: Wenn der Genitiv Singular (gegenüber den anderen Kasus) markiert ist, dann ausnahmslos mit $s$. Das $s$ ist ganz offensichtlich das Genitivzeichen par excellence, und das $s$ ist so stark, dass es sogar bei einigen schwachen benutzt wird, nämlich wiederum beim Typ Name. Wer also des Mohrs statt des Mohren, des Bärs statt des Bären sagt, der tut wiederum nichts anderes, als Mohr und Bär wie normale Substantive zu behandeln. Kann und soll man das verbieten?

Ein weiterer "Fehler", der bei der Flexion der schwachen Maskulina vorkommt und der bisher noch nicht erwähnt wurde, ist die Hinzufügung eines $s$ an das bereits vorhandene Genitivsuffix -en, wie in des Automatens (ein Hörbeleg). Kann auch dieser "Fehler" als systematisch "richtig" erklärt werden? Nun, wenn ein Sprecher neben des Automatens den Mensch und dem 
Bär sagt, dann kann man allenfalls konstatieren, dass der Genitiv wiederum auf das für den Genitiv unmarkierte $s$ auslautet. Eine große Zukunft wird man einem solchen Paradigma jedoch (mangels Vorbild in anderen Deklinationsklassen) nicht voraussagen können. Wenn der Sprecher aber, wie der Autor des Zitats in

(3) Sicherheitsdenken ist die älteste Überlebenstechnik des Menschens, nur haben wir heute mehr zu bedenken als Nüsse zu sammeln und möglichst einen großen Bären zu erlegen. (Die Welt, 03.01.2000, S. 31)

des Menschens neben den Bären sagt, dann folgt er der Regel, dass der Genitiv formal von Akkusativ und Dativ unterschieden werden muss. Und wenn er akzeptiert, dass Mensch und Bär schwach flektiert werden, dann bleibt für den Genitiv allein die Form Menschens oder Bärens. Und dass das nicht etwas völlig Neues und nie Dagewesenes ist, das zeigt wiederum der Typ Name.

Der Vergleich mit den anderen Deklinationsklassen zeigt also deutlich, dass das Flexionsmuster der schwachen Maskulina stark abweichend oder, wie Wurzel (1984) sagen würde, nicht strukturangemessen ist.

\section{3 "Anerkannte Unterlassung der Deklination" oder "nicht anerkannte unterbliebene Unterlassung der Deklination"?}

Die im vorigen Abschnitt herausgestellten Anomalien der schwachen Substantivflexion sind nahezu unbedeutend verglichen mit der größten Anomalie, die wir bei den schwachen Maskulina finden und die bisher in der Literatur und vor allem in den Grammatiken viel zu wenig Beachtung gefunden hat. Die schwachen Maskulina können nämlich - bei standardsprachlicher Verwendung - gar nicht immer mit Akkusativ- und Dativ-Suffix verwendet werden! Diesen Umstand hat schon Keseling (1968) in einem leider unbeachtet gebliebenen Aufsatz beleuchtet, und er wird auch in Wahrig (2003) und Duden (2001) erwähnt. Im Wahrig heißt es dazu:

Die Maskulina der schwachen Deklinationsklasse erhalten im Akkusativ und im Dativ Singular in der Regel dann keine Endung, wenn sie ohne Artikel, Pronomen oder Adjektiv stehen. Dies ist vor allem nach Präpositionen möglich:

der Unterschied zwischen Mensch und Affe; von Mensch zu Mensch

Obwohl die Präpositionen zwischen, von und zu den Dativ fordern, stehen hier die endungslosen Formen Mensch und Affe, da (aufgrund des fehlenden Artikels) die Form Menschen bzw. Affen als Plural interpretiert würde. (Wahrig 2003: 327)

Im Duden ist das (im Gegensatz zur bisher besprochenen "nicht anerkannten Unterlassung der Deklination") die "anerkannte Unterlassung" der Deklination. Die Beispiele dort sind:

Ich sag es Fürst und Edelmann; das Verhältnis zwischen Patient und Arzt; die Grenze zwischen Affe und Mensch; ein Forstmeister mit Assistent; am Wortende nach Konsonant (nicht: Konsonanten) spricht man ...; Das Gesuch muss Name (nicht: Namen), Beruf und Anschrift enthalten. (Duden 2001: 857, 858) 
Streng strukturalistisch betrachtet heißt das, dass die schwachen Maskulina zwei AkkusativSingular- und zwei Dativ-Singular-Formen haben, eine mit und eine ohne $n$-Suffix. Welche Form zu wählen ist, bestimmt allein die syntaktische Verwendung. Der Sprecher muss also, wenn er alles richtig machen will, bei der Verwendung eines schwachen Maskulinums immer entscheiden, welche der beiden Formen er zu wählen hat, und es kann sowohl die Form Mensch die falsche Form für den Dativ sein als auch die Form Menschen. Kann man es da einem Sprecher verdenken, wenn er sich entscheidet, das System zu vereinfachen und sich auf die Verwendung einer der beiden Formen, und zwar der suffixlosen (analog zu den anderen Deklinationsklassen) zu beschränken? Überspitzt könnte man formulieren, dass die Unterlassung der Deklination äußerst vernünftig ist und von einem hohen Grad von Einsicht in das Gesamtsystem der Substantivflexion des Deutschen bzw., wie Wurzel es ausdrücken würde, in die mangelnde Systemangemessenheit der schwachen Maskulina zeugt.

Übrigens stimme ich der Präskription, die der Duden hier vornimmt, ausdrücklich zu. Auch ich halte die Formen Konsonanten und Namen in den genannten Fällen für falsch, und sie scheinen mir ein viel schwerwiegenderer Regelverstoß zu sein als die (vom Duden) "nicht anerkannte Unterlassung der Deklination", für deren Anerkennung in Fällen wie den bisher besprochenen ich nachdrücklich plädieren möchte. Man sollte also auf Fehler vom Typ am Wortende nach Konsonanten [Singular] deutlicher und nachdrücklicher hinweisen, als dies im Duden (und auch im Wahrig) geschieht. Und statt von "anerkannter Unterlassung der Deklination" (was einen liberalen Klang hat und sich so anhört, als sei hier etwas nur erlaubt, nicht etwa geboten), sollte man von "nicht anerkannter unterbliebener Unterlassung der Deklination" (was einer "nicht anerkannten Deklination" gleichkommt) sprechen. Denn Fehler werden auch hier gemacht, wie die Belege in (14) bis (18) zeigen.

(14) Auf dem historischen Gipfel mit Südkoreas Präsidenten Kim Dae Jung in Pjöngjang überraschte der einst gefürchtete Dunkelmann die Welt als jovialer [...] Gastgeber.

(Der Spiegel 37/2000 S. 182)

(15) Gaddafi kämpft mit Südafrikas Präsidenten Thabo Mbeki nicht nur um die Führungsrolle in der neuen Afrikanischen Union, sondern vor allem um deren Ausrichtung.

(General-Anzeiger, Bonn, 18.07.2002, S. 2)

(16) Jetzt erst war ihm eingefallen, daß er Heinrich in Canossa nur als Christen, nicht aber als König vom Bann befreit hatte.

(Frankfurter Allgemeine Zeitung, 27.02.1999, S. IV)

(17) Rechts- und Staatswissenschaftliche Fakultät ehrt Präsidenten Venezuelas

(Bonner Universitäts-Nachrichten Nr. 210, Mai 1998, S. 25)

(18) Stichwahl entscheidet über Präsidenten in Nordzypern

(Die Welt, 17.04.2000, S. 7)

Dabei mag das Kasussuffix in (14) bis (16) noch eher hinnehmbar sein, da hier immerhin klar ist, um welchen Präsidenten bzw. Christ (!) es sich handelt. Dagegen können wir in (17) und (18) nur auf Grund unseres Weltwissens annehmen, dass es sich wohl jeweils um den Staatspräsidenten handelt und dass es demzufolge (da Staaten nur einen Präsidenten zu haben 
pflegen) wohl um genau einen Präsidenten handelt. Aber schon bei der Schlagzeile Stichwahl entscheidet über Präsidenten in Zypern wäre nicht einmal das eindeutig.

\section{$4 \quad$ Schluss}

In Abschnitt 3 habe ich einige Gründe aufgeführt, die dafür verantwortlich sein dürften, dass kompetente muttersprachliche Sprecher des Deutschen Zweifel bezüglich der Flexionsformen der schwachen Maskulina haben, und ich habe erläutert, warum es so häufig zu suffixlosen Akkusativ- und Dativformen (hingegen niemals zu suffixlosen Genitivformen!) kommt. Dabei darf allerdings nicht vergessen werden, dass die genannten Entwicklungen fast ausschließlich bei Substantiven vorkommen, die nicht auf Schwa enden. Die Maskulina auf Schwa bilden nämlich (im Gegensatz zum Typ Mensch/Bär und auch zu den ultimabetonten Mehrsilbern fremder Herkunft) eine homogene Gruppe: Alle auf Schwa auslautenden Maskulina flektieren schwach (einzige Ausnahmen: Käse und Substantive mit dem Wortbildungspräfix $\mathrm{Ge}$-); diese Substantive bilden also eine geschlossene, wohldefinierte Klasse, hier gibt es keine Abweichungen, und es kommt nicht zu Unsicherheiten. Die schwachen Maskulina auf Schwa werden (außer bei "nicht anerkannter Deklination"; vgl. 3.3.) immer flektiert, und hier kommt grundsätzlich keine "Unterlassung der Deklination" vor. Wirklich nie? Nun, man soll bekanntlich nie nie sagen. Vergleiche Beispiel (2).

\section{Literaturangaben}

Duden Bd. 9. (2001): Richtiges und gutes Deutsch. Wörterbuch der sprachlichen Zweifelsfälle. 5. Aufl. Mannheim etc.

Joeres, Rolf (1996): "Der Friede oder der Frieden. Ein Normproblem der Substantivflexion". Sprachwissenschaft 21: 301-336.

Keseling, Gisbert (1968): "'Irregularitäten' in der deutschen Substantivflexion". Zeitschrift für Mundartforschung 35: 233-243.

Köpcke, Klaus-Michael (1995): "Die Klassifikation der schwachen Maskulina in der deutschen Gegenwartssprache. Ein Beispiel für die Leistungsfähigkeit der Prototypentheorie". Zeitschrift für Sprachwissenschaft 14: 159-180.

Wahrig Bd. 5 (2003): Fehlerfreies und gutes Deutsch. Das zuverlässige Nachschlagewerk zur Klärung sprachlicher Zweifelsfälle. Gütersloh/München.

Wurzel, Wolfgang Ullrich (1984): Flexionsmorphologie und Natürlichkeit. Ein Beitrag zur morphologischen Theoriebildung. Berlin (= Studia grammatica XXI). 